\title{
Patterns of Recurrence After Salvage Radiotherapy Encompassing Pelvic Lymphatics in Men with High-risk Prostate Cancer
}

\author{
MINJI KOH ${ }^{1}$, YOUNG SEOK KIM ${ }^{1}$, HANJONG AHN $^{2}$ and CHOUNG-SOO KIM ${ }^{2}$ \\ ${ }^{1}$ Department of Radiation Oncology, Asan Medical Center, University of Ulsan, \\ College of Medicine, Seoul, Republic of Korea; \\ ${ }^{2}$ Department of Urology, Asan Medical Center, University of Ulsan, \\ College of Medicine, Seoul, Republic of Korea
}

\begin{abstract}
Background/Aim: The efficacy of adjuvant or salvage radiation to the regional lymph node area has not been fully investigated in high-risk prostate cancer patients; instead, radiotherapy is limited to the prostate fossa. The present study aimed to assess patterns of recurrence in prostate cancer patients with biochemical failure $(B C F)$ who were treated with whole-pelvic salvage radiotherapy (SRT) following radical prostatectomy. Patients and Methods: The clinical data from 196 high-risk prostate cancer patients who received SRT for BCF after radical prostatectomy were reviewed. BCF after SRT was detected in 80 patients, and 59 patients underwent imaging studies. Results: Twenty four recurrences in 16 patients were identified, including 13 bone metastases, 6 vesicourethral anastomosis site recurrences, and 5 lymph node recurrences (one simultaneous with vesicourethral anastomosis). Regarding the treatment field, no in-field nodal recurrence was observed, whereas 4 outof-field and 1 edge-of-field recurrences were detected. Conclusion: Locoregional recurrence was most common at the anastomosis site. Most nodal recurrences were located outside the pelvis, suggesting that elective pelvic nodal irradiation should be recommended in a selected patient population.
\end{abstract}

In the postprostatectomy setting, in either adjuvant or salvage radiotherapy (SRT), the radiotherapy (RT) field is traditionally limited to the prostate fossa (1-3), based on

Correspondence to: Young Seok Kim, Department of Radiation Oncology, 88, Olympic-ro 43-gil, Songpa-gu, Seoul 05505, Republic of Korea. Tel: +82 230105614, Fax: +82 230106950, e-mail: ysk@amc.seoul.kr

Key Words: Prostate cancer, salvage radiotherapy, pattern of failure, pelvic nodal irradiation. reports showing that the predominant site of failure is local (4). However, there is no definite consensus regarding radiation to the regional lymph node (LN) area, particularly in patients with high-risk prostate cancer. Several retrospective studies have reported on the efficacy of whole-pelvic RT (WPRT); Spiotto et al. (5) indicated that WPRT with androgen deprivation therapy (ADT) achieved superior biochemical failure (BCF)-free survival to that of prostate bed RT (PBRT) in high-risk patients. Moghanaki et al. (6) reported an improved biochemical control with WPRT only in patients with pre-SRT prostate-specific antigen (PSA) levels $>0.4 \mathrm{ng} / \mathrm{ml}$. In high-risk patients with $\mathrm{BCF}$ following radical prostatectomy (RP), the clinical outcomes following RT to the prostate fossa remain unsatisfactory, with a 5-year biochemical recurrence-free survival of $<50 \%(1,7-9)$. The efficacy of WPRT for improving the outcome of SRT is currently under evaluation in a Radiation Therapy Oncology Group (RTOG) 0534 phase 3 trial.

Understanding the anatomic patterns of failure after SRT might be helpful for evaluating the efficacy of SRT and determining the optimal dose and RT field. A recent study analyzing the pattern of failure after SRT to the prostate fossa reported that $12 \%$ of patients with BCF had in-field (IF) recurrence, whereas $88 \%$ had out-of-field (OOF) recurrence (10). In addition, $67 \%$ of the recurrences were nodal and located inferior to the aortic bifurcation level (10). Although this finding is merely hypothesis-generating, the recurrence patterns after the addition of elective nodal irradiation (ENI) could differ from those after PBRT.

The pattern of recurrence after elective pelvic LN SRT has not been described in detail. Whole-pelvic SRT has been used in the majority of high-risk prostate cancer patients in Asan Medical Center since 2007. Here, we retrospectively analyzed recurrence patterns to determine the optimal RT field in this patient population. 


\section{Patients and Methods}

Between 2007 and 2015, 196 consecutive patients with high-risk prostate cancer received SRT for BCF following RP at Asan Medical Center. The details on the SRT method were previously described (11). In brief, pretreatment evaluation included complete history, pelvic magnetic resonance imaging (MRI), bone scan, and laboratory tests including PSA measurements. All patients were treated with whole pelvic, intensity-modulated radiation therapy (WP-IMRT). Computed tomography (CT) simulation was performed in the supine position (GE Light Speed RT, GE Healthcare, Buckinghamshire, UK) using intravenous contrast agent. The clinical target volume included the prostate bed, the obturator, and the external, internal, and lower regions of the common iliac nodal basin. In general, the cranial margin was set at the level of the L5-S1 interface. The planning target volume (PTV) was delineated with anisotropic 3-7 mm extension to the clinical target volume. Inverse treatment planning of IMRT was performed with the Helios algorithm of the Eclipse planning system (Eclipse V10.0, Varian Medical System, Palo Alto, CA, USA). The irradiation doses and fractionation schedule during the treatment period were as follows: until 2010 , the whole pelvis and prostate bed were irradiated at 46 Gy and $20 \mathrm{~Gy}$, with 2 Gy per fraction, respectively $(n=20) ; 4$ Gy in two additional boost fractions were administered in locally recurrent lesions based on imaging studies before SRT $(n=38)$, except in two patients treated with the simultaneous integrated boost technique. After 2011, whole pelvic and boost doses were 44 Gy and 22 Gy with a fraction size of 2.2 Gy $(n=126)$, respectively, and $6.6 \mathrm{~Gy}$ in three boost fractions were administered in cases of overt locally recurrent lesions $(n=10)$. An endorectal balloon was used to reduce the irradiated rectal volume and improve reproducibility during the course of SRT after 2009 (12). All patients were examined at least once a week during the course of SRT. After completion of SRT, patients were evaluated by a radiation oncologist and an urologist every 3 months for 2 years, and at least every 6 months thereafter. BCF after RP and SRT was defined as a PSA level $>0.2 \mathrm{ng} / \mathrm{ml}$ and another confirmatory rise. The present study was approved by the institutional review board of Asan Medical Center, and the requirement for informed consent was waived.

Flow chart of the enrollment of the study population is shown in Figure 1. During the median follow-up period of 74 months, 80 of 196 patients experienced a second BCF despite SRT. Of them, 59 patients $(74 \%)$ were evaluated using various imaging modalities including bone scan $(n=54)$, CT $(n=46)$, MRI $(n=22)$, and ${ }^{18} \mathrm{~F}$-fluorodeoxyglucose positron-emission tomography/CT (F18-FDG PET/CT) $(n=8)$ at the attending physician's discretion. All images were reviewed by certified radiologists, and 24 visible recurrences were detected in 16 patients. Recurrences were classified as bone metastasis or locoregional recurrences. No organ metastasis was detected. Locoregional recurrence was further categorized as follows: IF, edge-of-field (EOF), and OOF, which was defined as any recurrence in the PTV, out of the PTV but included within a $2 \mathrm{~cm}$ extension to the PTV, and outside the $2 \mathrm{~cm}$ extension to the PTV.

The $t$-test and Chi-square test were used to evaluate related factors between two groups; $p$-values of $<0.05$ were considered statistically significant. IBM ${ }^{\circledR}$ SPSS ${ }^{\circledR}$ for Windows, version 22.0 was used for the statistical analysis (IBM Corporation, Armonk, NY, USA).

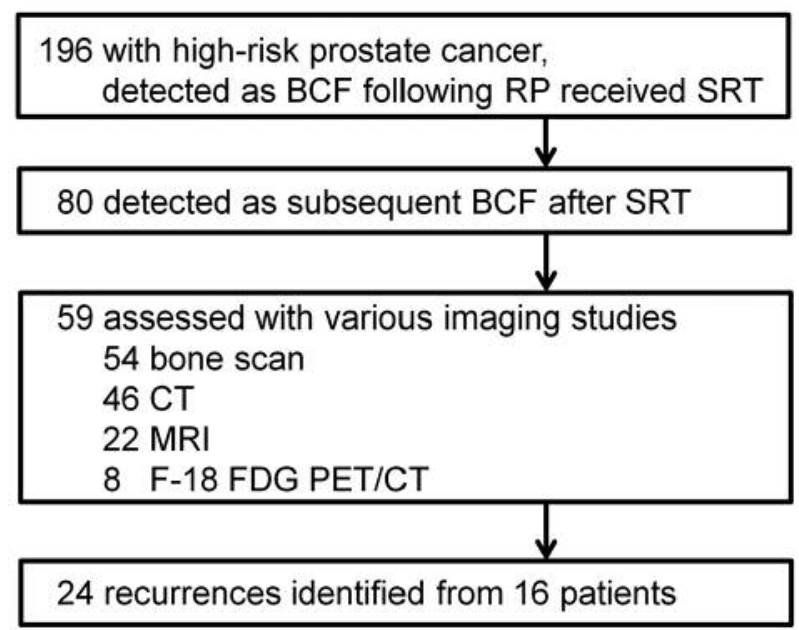

Figure 1. Enrollment of study patients. BCF, Biochemical failure; $R P$, radical prostatectomy; $S R T$, salvage radiotherapy; $C T$, computed tomography; MRI, magnetic resonance imaging; F-18 FDG PET/CT, positron emission tomography with 2-deoxy-2-[fluorine-18]fluoro-Dglucose integrated with computed tomography.

\section{Results}

The clinical and pathological characteristics of 59 patients evaluated using various imaging modalities for the detection of BCF following SRT are described in Table I. In these patients, 24 sites of recurrence were identified in 16 men. The sites of recurrence are summarized in Table II. The most common recurrence was bone metastasis; 13 metastatic bony lesions were identified in six patients. Two patients showed multiple osseous recurrences in more than two separate lesions, including the rib, vertebra, femur, and cranium. Other sites of osseous recurrence were the L1 spine and the pelvic bone. Excluding bony metastases, the most common site of recurrence was the vesicourethral anastomosis (VUA) site. Six anastomotic recurrences were detected in six men. Four patients experienced LN recurrence only, and one showed simultaneous VUA and nodal failure.

The sites of recurrence superimposed on digitally reconstructed radiography (DRR) are shown in Figure 2. In all cases of VUA recurrence, the site was included in the prostate bed boost volume, which was irradiated at 66 to 70 Gy in the previous SRT. Nodal failure sites were located at the common iliac $(n=2)$ or para-aortic $\mathrm{LN}$ area $(n=3)$, and no IF nodal recurrence was observed. One nodal failure occurred at EOF, within the $2 \mathrm{~cm}$ extension to the PTV. The characteristics of five patients with nodal recurrence are described in Table III. A high Gleason score $\geq 8$, seminal vesicle invasion, and pre-SRT PSA $\geq 1$ were identified in five, four, and three men, respectively. 
Table I. Patient characteristics.

\begin{tabular}{lc}
\hline Variables & No. of patients $(\%)$ \\
\hline Age (year) & \\
Median (range) & $64(48-75)$ \\
Initial PSA level (ng/ml) & \\
$\quad$ Median (IQR) & $21.4(10.8-42.2)$ \\
Gleason score & \\
7 (3+4) & $7(11.9)$ \\
7 (4+3) & $11(18.6)$ \\
$8-10$ & $41(69.5)$ \\
pT stage & \\
pT2a-2c & $12(20.3)$ \\
pT3a & $19(32.2)$ \\
pT3b-4 & $28(47.5)$ \\
pN stage & \\
Negative & $52(88.1)$ \\
Positive & $7(11.9)$ \\
No. of harvested lymph nodes & $6(3-8)$ \\
Median (IQR) & \\
Time to 1st BCF from RP (months) & $9(1-95)$ \\
Median (range) & \\
Roach score for LNI & \\
Median (IQR) & \\
36.4 (25.2-53.3) & \\
PSA level before SRT (ng/ml) & \\
Median (IQR) & \\
EQD2 ( $\alpha / \beta$ of 2 Gy) & \\
Median (range) & \\
No. of fractions & \\
Median (range) & \\
Pre-RT ADT & \\
Time to BCF after SRT (months) & \\
Median (range) & \\
\hline
\end{tabular}

PSA, Prostate-specific antigen; IQR, interquartile range; BCF, biochemical failure; RP, radical prostatectomy; LNI, lymph node involvement; SRT, salvage radiotherapy; RT, radiotherapy; EQD2, equivalent dose in 2 Gy fractions; ADT, androgen deprivation therapy.

Patients with clinically manifested recurrence(s) were compared with those with BCF only without obvious recurrence in terms of clinical and pathological features (Table IV). Statistically significant differences between the two groups were observed in PSA level before SRT $(p=0.045)$ in the $t$-test and ADT before SRT $(p<0.01)$ in the Chi-square test.

\section{Discussion}

In the present study, analysis of the patterns of failure in high-risk prostate cancer patients who received SRT encompassing the pelvic $\mathrm{LN}$ area showed that osseous metastasis was the most common recurrence followed by VUA recurrence. One surprising finding was that there were no patients with IF nodal recurrence, suggesting that elective pelvic nodal irradiation should be recommended in a selected

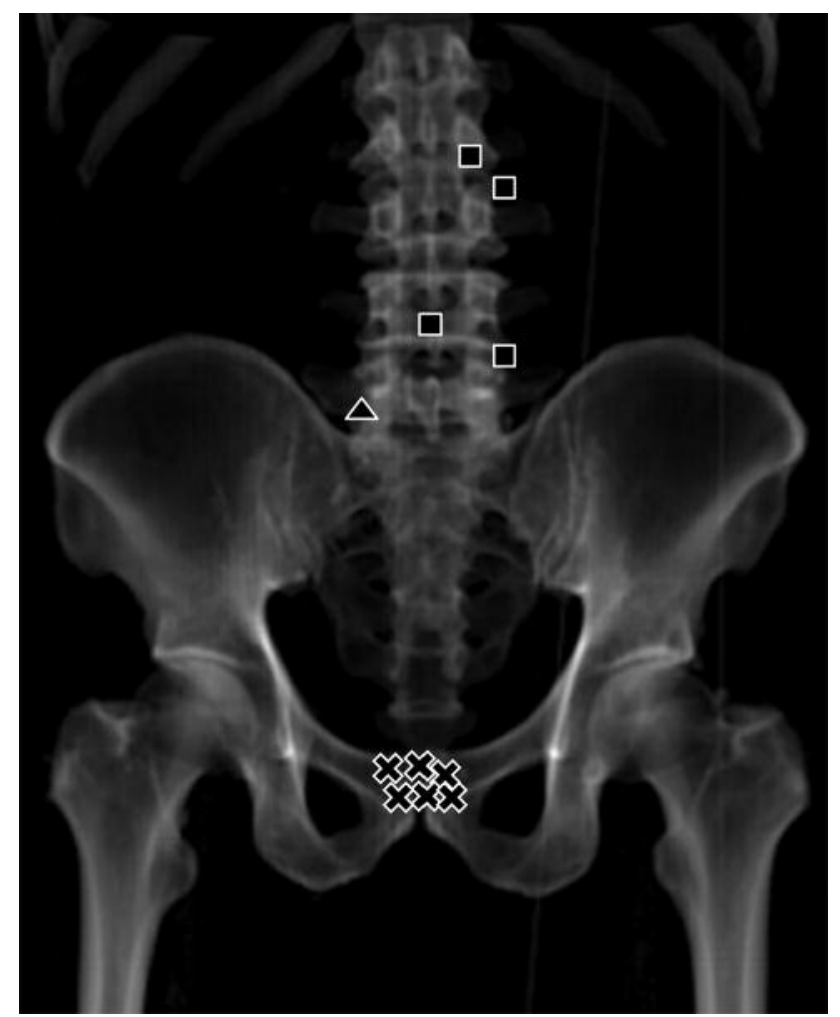

Figure 2. Recurrence sites superimposed in an anteroposterior view. The sites of recurrence are indicated as follows: nodal recurrence out of field (ם), nodal recurrence edge of field ( $\mathbf{\Delta})$, vesicourethral anastomosis site recurrence in the prostate bed boost field $(\times)$.

Table II. Characterization of recurrence sites

\begin{tabular}{lccc}
\hline Site of recurrence & $\begin{array}{c}\text { Field of } \\
\text { recurrence }\end{array}$ & $\begin{array}{c}\text { No. of } \\
\text { lesions (\%) }\end{array}$ & $\begin{array}{c}\text { No. of } \\
\text { patients (\%) }\end{array}$ \\
\hline $\begin{array}{l}\text { Vesicourethral anastomosis } \\
\text { Lymph node }\end{array}$ & IF & $6^{\mathrm{a}(25)}$ & $6(37.5)$ \\
Right CILN & EOF & $\begin{array}{c}5(20.8) \\
1(4.2)\end{array}$ & $\begin{array}{l}5(31.3) \\
1(6.3)\end{array}$ \\
Para-aortic LN & OOF & $3(12.5)$ & $3(18.8)$ \\
Left CILN & OOF & $1^{\mathrm{a}(4.2)}$ & $1(6.3)$ \\
Bone & & $13(54.2)$ & $6(37.5)$ \\
Total & & 24 & 16 \\
\hline
\end{tabular}

IF, In field; CILN, common iliac lymph node; EOF, edge of field $(2 \mathrm{~cm}$ periphery to PTV); OOF, out of field. aOne patient with combined vesicourethral anastomosis and left CILN recurrence.

group of patients. Patients with clinically manifested recurrences had a significantly higher level of pre-SRT PSA than those who experienced BCF without obvious recurrences. As this finding is hypothesis-generating only, it should be interpreted with caution. 
Table III. Description of patients with nodal recurrence $(n=5)$.

\begin{tabular}{|c|c|c|c|c|c|}
\hline Variables & $\# 1$ & $\# 2$ & \#3 & $\# 4$ & \#5 \\
\hline Age (years) & 56 & 57 & 70 & 63 & 66 \\
\hline Initial PSA level (ng/ml) & 544 & 40 & 40.1 & 7.55 & 6.1 \\
\hline Gleason score & 8 & 9 & 9 & 9 & 8 \\
\hline pT stage & $3 \mathrm{~b}$ & $3 \mathrm{~b}$ & $2 \mathrm{c}$ & $3 b$ & $3 b$ \\
\hline $\mathrm{pN}$ stage & 0 & 0 & 0 & 0 & 0 \\
\hline No. of harvested lymph nodes & 5 & 2 & 4 & 2 & 8 \\
\hline Time to 1st BCF from RP (months) & 4 & 3 & 26 & 7 & 16 \\
\hline Roach score for LNI & 100 & 56.7 & 56.7 & 35.0 & 24.1 \\
\hline PSA level before SRT $(\mathrm{ng} / \mathrm{ml})$ & 3.1 & 2.8 & 1 & 0.55 & 0.67 \\
\hline ADT use before SRT & + & - & + & + & + \\
\hline Time to BCF from SRT (months) & 5 & 8 & 24 & 79 & 49 \\
\hline Site of recurrence & Right CILN & PALN & Left CILN, anastomosis & PALN & PALN \\
\hline
\end{tabular}

PSA, Prostate-specific antigen; BCF, biochemical failure; RP, radical prostatectomy; LNI, lymph node involvement; SRT, salvage radiotherapy; ADT, androgen deprivation therapy; CILN, common iliac lymph node; PALN, para-aortic lymph node.

Regarding the radiation dose for SRT following RP, the optimal dose remains to be clearly defined. A dose of 64-72 Gy in standard fractionation for adjuvant/salvage postprostatectomy RT is recommended (13). Retrospective data suggest the presence of a dose response of SRT after RP $(14,15)$, supporting a SRT dose above 66-70 Gy. In addition, the application of IMRT allows a safe dose escalation to 76 Gy to the prostate bed (16). A recent meta-analysis identified a $2.0 \%$ gain in relapse-free survival for each additional Gy (14). As the main failure site is localized around the VUA site, dose escalation to this area can be considered as an option to improve the local control rate. A phase III randomized trial of MRImapped dose-escalated SRT is in progress, with escalating radiation doses up to 81.3 Gy using a simultaneous incorporated hypofractionated boost (17).

The elective inclusion of the nodal area is another issue lacking a defined consensus. The RT field is usually limited to the prostate fossa (1-3), and the inclusion of ENI has recently been addressed. Retrospective studies showed an improvement in biochemical control following whole pelvic SRT $(5,6,18)$. One of the reasons for the reluctance in irradiating the pelvic node area is the possibility of side effects. In recent studies, the toxicity of WP-IMRT was lower in men treated with definitive (19) and salvage RT (11, 20) than in those treated with 3-dimensional conformal RT. A comparative study reported that WP-IMRT only increased acute gastrointestinal toxicities without affecting genitourinary or late gastrointestinal toxicities compared with prostate bed IMRT (21). Therefore, WP-IMRT could be considered as a potential alternative to improve disease control with allowable levels of toxicity.

In the current study, all patients underwent pelvic LN dissection (PLND), except for one patient who received RP in another institution. Dissected LNs were usually confined to the obturator and internal iliac node area, and were evaluated only by hematoxylin and eosin (H\&E) staining. The addition of extended PLND and immunohistochemical (IHC) staining resulted in the detection of LN metastasis in $26 \%$ of patients with clinically localized prostate cancer (22). The frequency of $\mathrm{LN}$ metastases increases to $61 \%$ in patients with certain risk factors such as a Gleason score $\geq 7$ and PSA $\geq 10.6 \mathrm{ng} / \mathrm{ml}$ (22). These risk factors were in line with most of the patients enrolled in the current study, and only $11.9 \%$ of the patients who underwent post-BCF imaging studies were diagnosed with a positive $\mathrm{N}$ stage. The possibility of undetected LN metastases needs to be considered in the patients treated in our institution, because extended PLND was not performed, and a small number of LNs were dissected and evaluated by H\&E staining alone. Therefore, the benefit of WPRT in the present study might be exaggerated to some extent.

Recently developed imaging modalities such as C-11 Choline PET (CholPET) or Ga-68 prostate-specific membrane antigen (PSMA) PET are being investigated for the early detection of recurrent lesions. Parker et al. (10) reported patterns of recurrence after PBRT by CholPET, and $88 \%$ of the identified recurrences were nodal failures, compared with $4 \%$ of recurrences limited to the prostatic fossa. In addition, $43 \%$ and $63 \%$ of nodal recurrences were within the RT field below the L5-S1 interface and the aortic bifurcation site, respectively (10). A study that evaluated patients with BCF after RT using Ga-68 PSMA for potential SRT reported that $27 \%, 14.3 \%, 4.3 \%$, and $8.6 \%$ of the patients had recurrent lesions in the prostatic fossa, in the pelvic nodes, in both the fossa and pelvic nodes, and outside the pelvis, respectively (23). These results suggest the possibility of microscopic recurrence lesions after RP, and a considerable number of these are located in the pelvic LN area. Schiller et al. used Ga-68 PSMA PET for SRT planning, and detected suspicious recurrent lesions in $87 \%$ 
Table IV. Comparison between patients with clinically manifested recurrence and those with only $B C F$.

\begin{tabular}{|c|c|c|c|}
\hline \multirow[t]{2}{*}{ Variables } & \multicolumn{2}{|c|}{ No. of patients (\%) } & $p$-Value \\
\hline & $\begin{array}{l}\text { Clinically } \\
\text { manifested } \\
\text { recurrence } \\
(n=16)\end{array}$ & $\begin{array}{c}\text { Only } \\
\text { BCF } \\
(n=43)\end{array}$ & \\
\hline
\end{tabular}

\begin{tabular}{|c|c|c|c|}
\hline \multicolumn{4}{|l|}{ Age (years) } \\
\hline Median (range) & $63(55-74)$ & $64(48-75)$ & 0.81 \\
\hline \multicolumn{4}{|c|}{ Initial PSA level (ng/ml) } \\
\hline Median (IQR) & $19.9(13.6-40.1)$ & $21.6(10.3-53.3)$ & 0.45 \\
\hline \multicolumn{4}{|l|}{ Gleason score } \\
\hline 7 & $2(12.5)$ & $16(37.2)$ & \multirow[t]{2}{*}{0.11} \\
\hline $8-10$ & $14(87.5)$ & $27(62.8)$ & \\
\hline \multicolumn{4}{|l|}{ pT stage } \\
\hline pT2a-3a & $7(43.8)$ & $24(55.8)$ & \multirow[t]{2}{*}{0.41} \\
\hline pT3b-4 & $9(56.3)$ & $19(44.2)$ & \\
\hline \multicolumn{4}{|l|}{ pN stage } \\
\hline Negative & $16(100)$ & $36(83.7)$ & \multirow[t]{2}{*}{0.17} \\
\hline Positive & $0(0)$ & $7(16.3)$ & \\
\hline \multicolumn{4}{|l|}{$\begin{array}{l}\text { No. of harvested } \\
\text { lymph nodes }\end{array}$} \\
\hline Median (IQR) & $5(2-8)$ & $6(4-9)$ & 0.13 \\
\hline \multicolumn{4}{|l|}{ Time to 1 st BCF from } \\
\hline \multicolumn{4}{|l|}{ RP (months) } \\
\hline Median (range) & $15(2-67)$ & $9(1-95)$ & 0.48 \\
\hline \multicolumn{4}{|l|}{ Roach score for LNI } \\
\hline Median (IQR) & $41.2(32.6-56.7)$ & $35.7(24.7-52.8)$ & 0.32 \\
\hline \multicolumn{4}{|l|}{ PSA level before } \\
\hline \multicolumn{4}{|l|}{ SRT (ng/ml) } \\
\hline Median (IQR) & $1.2(0.6-2.6)$ & $0.7(0.4-0.9)$ & 0.05 \\
\hline \multicolumn{4}{|l|}{$\mathrm{EQD} 2(\alpha / \beta$ of $2 \mathrm{~Gy})$} \\
\hline Median (range) & $69.3(64-70)$ & $69.3(66-70)$ & 0.70 \\
\hline ADT use before SRT & $13(81.3)$ & $13(30.2)$ & $<0.00$ \\
\hline \multicolumn{4}{|l|}{ Time to BCF after } \\
\hline \multicolumn{4}{|l|}{ SRT (months) } \\
\hline Median (range) & $24(4-84)$ & $24(3-82)$ & 0.74 \\
\hline
\end{tabular}

PSA, Prostate-specific antigen; IQR, interquartile range; $\mathrm{BCF}$, biochemical failure; RP, radical prostatectomy; LNI, lymph node involvement; SRT, salvage radiotherapy; EQD2, equivalent dose in 2 Gy fraction; ADT, androgen deprivation therapy.

of the cases that were not detected by conventional imaging including CT and MRI (24). In addition, $40 \%$ of the detected recurrent LNs were not included in the standard whole pelvic radiation field (24). The outcome of PSMA PET-based SRT planning is favorable, showing a significant response during the median follow-up of 20 months; the median post-RT PSA level was $0.07 \mathrm{ng} / \mathrm{ml}$ and $81 \%$ of the patients had a PSA level $\leq 0.2 \mathrm{ng} / \mathrm{ml}$ (25). Further efforts using advanced imaging modalities to modify the treatment volume could be considered to improve the outcome of SRT.

The current study had several limitations, including its retrospective nature and the small number of patients included, which may have introduced potential bias. In addition, not all patients showing BCF after SRT underwent imaging studies, and various imaging modalities were used without consistent guidelines, although a bone scan was performed in most patients. All cases with non-osseous recurrences were assessed using MRI and/or CT, which have a low sensitivity for detecting LN involvement (26). Advanced imaging modalities with a higher sensitivity for detecting recurrent lesions may have allowed for a more detailed definition of the pattern of recurrence. Despite these shortcomings, the present study has several unique attributes as follows: i) the patient population was homogeneous, as all patients were diagnosed with highrisk prostate cancer; ii) the SRT technique and the definition of BCF after SRT were consistent, as the study was performed in a single institution; iii) an intriguing and hypothesisgenerating finding was that IF nodal recurrence was not detected when whole-pelvic SRT was implemented; and iv) the present study is the first report describing recurrence patterns after whole pelvic SRT for BCF following RP.

In conclusion, most locoregional recurrences occurred at the anastomosis site, suggesting the need for dose escalation. Most nodal recurrences were located outside the pelvis, suggesting that elective pelvic node irradiation should be considered in a selected group of patients. A well-designed, prospective study is warranted to confirm these findings.

\section{Acknowledgements}

This work was supported by the National Research Foundation of Korea (NRF) grant funded by the Korea government (MSIP) [NRF2016R1C1B2012823].

\section{References}

1 Stephenson AJ, Shariat SF, Zelefsky MJ, Kattan MW, Butler EB, Teh BS, Klein EA, Kupelian PA, Roehrborn CG, Pistenmaa DA, Pacholke HD, Liauw SL, Katz MS, Leibel SA, Scardino PT and Slawin KM: Salvage radiotherapy for recurrent prostate cancer after radical prostatectomy. JAMA 291: 1325-1332, 2004.

2 Pfister D, Bolla M, Briganti A, Carroll P, Cozzarini C, Joniau S, van Poppel H, Roach M, Stephenson A, Wiegel T and Zelefsky MJ: Early salvage radiotherapy following radical prostatectomy. Eur Urol 65: 1034-1043, 2014.

3 Wiegel T, Bartkowiak D, Bottke D, Bronner C, Steiner U, Siegmann A, Golz R, Storkel S, Willich N, Semjonow A, Stockle M, Rube C, Rebmann U, Kalble T, Feldmann HJ, Wirth M, Hofmann R, Engenhart-Cabillic R, Hinke A, Hinkelbein W and Miller K: Adjuvant radiotherapy versus wait-and-see after radical prostatectomy: 10-year follow-up of the ARO 9602/AUO AP 09/95 trial. Eur Urol 66: 243-250, 2014.

4 Swanson GP, Hussey MA, Tangen CM, Chin J, Messing E, Canby-Hagino E, Forman JD, Thompson IM and Crawford ED: Predominant treatment failure in postprostatectomy patients is local: analysis of patterns of treatment failure in SWOG 8794. J Clin Oncol 25: 2225-2229, 2007. 
5 Spiotto MT, Hancock SL and King CR: Radiotherapy after prostatectomy: improved biochemical relapse-free survival with whole pelvic compared with prostate bed only for high-risk patients. Int J Radiat Oncol Biol Phys 69: 54-61, 2007.

6 Moghanaki D, Koontz BF, Karlin JD, Wan W, Mukhopadhay N, Hagan MP and Anscher MS: Elective irradiation of pelvic lymph nodes during postprostatectomy salvage radiotherapy. Cancer 119: 52-60, 2013.

7 Brooks JP, Albert PS, Wilder RB, Gant DA, McLeod DG and Poggi MM: Long-term salvage radiotherapy outcome after radical prostatectomy and relapse predictors. J Urol 174: 2204$2208,2005$.

8 van Dessel LF, Reuvers SHM, Bangma CH and Aluwini S: Salvage radiotherapy after radical prostatectomy: long-term results of urinary incontinence, toxicity and treatment outcomes. Clin Transl Radiat Oncol 11: 26-32, 2018.

9 Buskirk SJ, Pisansky TM, Schild SE, Macdonald OK, Wehle MJ, Kozelsky TF, Collie AC, Ferrigni RG, Myers RP, Prussak KA, Heckman MG, Crook JE, Parker AS and Igel TC: Salvage radiotherapy for isolated prostate specific antigen increase after radical prostatectomy: evaluation of prognostic factors and creation of a prognostic scoring system. J Urol 176: 985-990, 2006.

10 Parker WP, Evans JD, Stish BJ, Park SS, Olivier K, Choo R, Nathan MA, Welch BT, Karnes RJ, Mynderse LA, Pisansky TM, Kwon ED, Lowe VJ and Davis BJ: Patterns of recurrence after postprostatectomy fossa radiation therapy identified by $\mathrm{C}-11$ choline positron emission tomography/computed tomography. Int J Radiat Oncol Biol Phys 97: 526-535, 2017.

11 Byun SJ, Kim YS, Ahn H and Kim CS: Image-guided, wholepelvic, intensity-modulated radiotherapy for biochemical recurrence following radical prostatectomy in high-risk prostate cancer patients. PLoS One 13: e0190479, 2018.

12 Joo JH, Kim YJ, Kim YS, Cho YP, Lee HY, Jeong CY, Kwak J and Cho BC: Analysis of prostate bed motion using an endorectal balloon and cone beam computed tomography during postprostatectomy radiotherapy. Onco Targets Ther 9: 3095$3100,2016$.

13 National Comprehensive Cancer Network: NCCN clinical practice guidelines in oncology (NCCNGuideline) Prostate cancer [Internet]. National Comprehensive Cancer Network. 2018. [Accessed August 29, 2018]. Available from: https:// www.nccn.org/professionals/physician_gls/f_guidelines.asp\#site

14 King CR: The dose-response of salvage radiotherapy following radical prostatectomy: a systematic review and meta-analysis. Radiother Oncol 121: 199-203, 2016.

15 Pisansky TM, Agrawal S, Hamstra DA, Koontz BF, Liauw SL, Efstathiou JA, Michalski JM, Feng FY, Abramowitz MC, Pollack A, Anscher MS, Moghanaki D, Den RB, Stephans KL, Zietman AL, Lee WR, Kattan MW, Stephenson AJ and Tendulkar RD: Salvage radiation therapy dose response for biochemical failure of prostate cancer after prostatectomy-a multi-institutional observational study. Int J Radiat Oncol Biol Phys 96: 1046-1053, 2016.

16 Ost P, Lumen N, Goessaert AS, Fonteyne V, De Troyer B, Jacobs F and De Meerleer G: High-dose salvage intensity-modulated radiotherapy with or without androgen deprivation after radical prostatectomy for rising or persisting prostate-specific antigen: 5-year results. Eur Urol 60: 842-849, 2011.
17 U.S. National Library of Medicine: A phase III randomized trial of MRI-mapped dose-escalated salvage radiotherapy postprostatectomy: The MAPS trial (MAPS) [Internet]. ClinicalTrials.gov. 2011. [Accessed August 29, 2018]. Available from: https://clinicaltrials.gov/ct2/show/NCT01411345

18 Song C, Kang HC, Kim JS, Eom KY, Kim IA, Chung JB, Hong SK, Byun SS and Lee SE: Elective pelvic versus prostate bedonly salvage radiotherapy following radical prostatectomy: a propensity score-matched analysis. Strahlenther Onkol 191: 801$809,2015$.

19 Muller AC, Lutjens J, Alber M, Eckert F, Bamberg M, Schilling D, Belka C and Ganswindt U: Toxicity and outcome of pelvic IMRT for node-positive prostate cancer. Strahlenther Onkol 188: 982-989, 2012

20 Alongi F, Fiorino C, Cozzarini C, Broggi S, Perna L, Cattaneo GM, Calandrino R and Di Muzio N: IMRT significantly reduces acute toxicity of whole-pelvis irradiation in patients treated with post-operative adjuvant or salvage radiotherapy after radical prostatectomy. Radiother Oncol 93: 207-212, 2009.

21 Deville C, Vapiwala N, Hwang WT, Lin H, Ad VB, Tochner Z and Both S: Comparative toxicity and dosimetric profile of whole-pelvis versus prostate bed-only intensity-modulated radiation therapy after prostatectomy. Int $\mathrm{J}$ Radiat Oncol Biol Phys 82: 1389-1396, 2012.

22 Heidenreich A, Varga Z and Von Knobloch R: Extended pelvic lymphadenectomy in patients undergoing radical prostatectomy: high incidence of lymph node metastasis. J Urol 167: 16811686, 2002.

23 van Leeuwen PJ, Stricker P, Hruby G, Kneebone A, Ting F, Thompson B, Nguyen Q, Ho B and Emmett L: (68) Ga-PSMA has a high detection rate of prostate cancer recurrence outside the prostatic fossa in patients being considered for salvage radiation treatment. BJU Int 117: 732-739, 2016.

24 Schiller K, Sauter K, Dewes S, Eiber M, Maurer T, Gschwend J, Combs SE and Habl G: Patterns of failure after radical prostatectomy in prostate cancer - implications for radiation therapy planning after (68)Ga-PSMA-PET imaging. Eur J Nucl Med Mol Imaging 44: 1656-1662, 2017.

25 Schmidt-Hegemann NS, Fendler WP, Ilhan H, Herlemann A, Buchner A, Stief C, Eze C, Rogowski P, Li M, Bartenstein P, Ganswindt U and Belka C: Outcome after PSMA PET/CT based radiotherapy in patients with biochemical persistence or recurrence after radical prostatectomy. Radiat Oncol 13: 37, 2018.

26 Torabi M, Aquino SL and Harisinghani MG: Current concepts in lymph node imaging. J Nucl Med 45: 1509-1518, 2004. 